International Journal of Pure and Applied Mathematics

Volume 97 No. 1 2014, 31-35

ISSN: 1311-8080 (printed version); ISSN: 1314-3395 (on-line version)

url: http://www.ijpam.eu

doi: http://dx.doi.org/10.12732/ijpam.v97i1.4

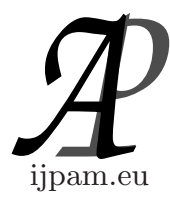

\title{
GRADED SUBMODULES WITH PSEUDO IRREDUCIBLE, PSEUDO PRIME AND STRICTLY NON-PRIME COMPONENTS
}

\author{
Rashid Abu-Dawwas ${ }^{1 \S}$, Khaldoun Al-Zoubi ${ }^{2}$ \\ ${ }^{1}$ Department of Mathematics \\ Yarmouk University \\ Irbid, JORDAN \\ ${ }^{2}$ Department of Mathematics and Statistics \\ Jordan University of Science and Technology \\ Irbid, JORDAN
}

\begin{abstract}
Let $G$ be a group. Let $R$ be a commutative $G$-graded ring, $M$ be a graded $R$-module and $N$ be a graded $R$-submodule of $M$. In this paper, we study some cases when $R$ is strongly graded ring and the component $N_{e}$ of $N$ is strictly non-prime, pseudo prime or pseudo irreducible $R_{e}$-submodule. For example, we prove that if $R$ is strongly graded, the components of $M$ are multiplication and $N_{e}$ is pseudo irreducible, then $N_{g}$ is pseudo prime for all $g \in G$.
\end{abstract}

AMS Subject Classification: 13A02, 16W50

Key Words: graded modules, pseudo prime graded submodules, multiplication modules

\section{Introduction}

A ring $R$ with unity 1 graded by a group $G$ will means that $R=\bigoplus_{g \in G} R_{g}$

Received: June 5, 2014

(C) 2014 Academic Publications, Ltd.

${ }^{\S}$ Correspondence author url: www.acadpubl.eu 
where $R_{g}$ is an additive subgroup of $R$ and $R_{g} R_{h} \subseteq R_{g h}$ for all $g, h \in G$. If the inclusion is an equality, then the ring is called strongly graded. Clearly, $R_{e}$ is a subring of $R$ with $1 \in R_{e}$. An $R$-module is said to be graded if $M=\bigoplus_{g \in G} M_{g}$ for a family of subgroups $\left\{M_{g}\right\}_{g \in G}$ of $M$ such that $R_{g} M_{h} \subseteq M_{g h}$ for all $g, h \in G$. Clearly, $M_{g}$ is an $R_{e}$-module for all $g \in G$. In a similar way, we define a strongly graded module. The ring $R$ is strongly graded if and only if every graded $R$ module is strongly graded. An $R$-submodule $N$ of a graded $R$-module $M$ is said to be graded $R$-submodule of $M$ if $N=\bigoplus_{g \in G}\left(N \cap M_{g}\right)$. For more details, we refer the readers to [4], as well as [5].

An $R$-module is said to be multiplication if for any $R$-submodule $N$ of $M$, $N=I M$ for some ideal $I$ of $R$. One can easily prove that if $M$ is multiplication, then $N=(N: M) M$ for every $R$-submodule $N$ of $M$ where $(N: M)=\{r \in$ $R: r M \subseteq N\}$ that is an ideal of $R$. In [1], Abd El-Bast and Smith have shown that $N$ is prime submodule if and only if $(N: M)$ is prime ideal provided that $M$ is multiplication. Let $N, K$ be two $R$-submodules of multiplication module $M$. Then $N=I M$ and $K=J M$ for some ideals $I, J$ of $R$ and then the multiplication $N K$ is defined by Ameri in [2] as $N K=(I M)(J M)=I J M$.

In this paper, we study graded submodules with multiplication components for pseudo prime and pseudo irreducible properties. Pseudo irreducible ideals and pseudo prime ideals are defined by McAdam and Swan in [3]. An ideal $I$ of a ring $R$ is said to be pseudo irreducible if one can not write $I=J K$ with $J+K=R$ where $I \neq R, J \neq R$ are ideals of $R$. Also, $I$ is said to be pseudo prime if whenever $J+K=R$ with $J K \subseteq I$, then either $J \subseteq I$ or $K \subseteq I$ where $I, J$ are ideals of $R$. Similarly, an $R$-submodule $N$ of a multiplication $R$-module $M$ is said to be pseudo irreducible if whenever $N=U V$ for some $R$-submodules $U, V$ of $M$ such that $U+V=M$, then either $U=M$ or $V=M$. Also, $N$ is said to be pseudo prime if whenever $U, V$ are $R$-submodules of $M$ such that $U V \subseteq N$ and $U+V=M$, then either $U \subseteq N$ or $V \subseteq N$.

Throughout this paper, unless stated otherwise, $R$ is commutative nontrivially graded ring.

\section{Results}

In this section, we introduce and prove the main results of the paper.

Lemma 2.1. Let $R$ be a $G$-graded ring and $M$ be graded $R$-module. If $N$ is a graded $R$-module of $M$, then $R_{g} N_{h} \subseteq N_{g h}$ for all $g, h \in G$.

Proof. Let $g, h \in G$. Then $R_{g} N_{h} \subseteq R_{g} M_{h} \subseteq M_{g h}$. On the other hand, 
$R_{g} N_{h} \subseteq R N=N$ and hence, $R_{g} N_{h} \subseteq N \bigcap M_{g h}=N_{g h}$.

Theorem 2.2. Let $R$ be a strongly $G$-graded ring and $M$ be a graded $R$-module. Suppose that $M_{e}$ is faithful multiplication $R_{e}$-module and $N$ is a graded $R$-submodule of $M$. If $N_{e}$ is pseudo irreducible $R_{e}$-submodule of $M_{e}$, then $\left(N_{g}: M_{g}\right)$ is pseudo irreducible ideal of $R_{e}$ for all $g \in G$.

Proof. Let $g \in G$ and suppose that $\left(N_{g}: M_{g}\right)=A B$ where $A$ and $B$ are two ideals of $R_{e}$ satisfy $A+B=R_{e}$. Then $A B M_{e}=A B R_{g^{-1}} M_{g}=R_{g^{-1}} A B M_{g} \subseteq$ $R_{g^{-1}} N_{g} \subseteq N_{e}$, i.e., $A B \subseteq\left(N_{e}: M_{e}\right)$. Let $r \in\left(N_{e}: M_{e}\right)$. Then $r M_{g}=r R_{g} M_{e}=$ $R_{g} r M_{e} \subseteq R_{g} N_{e} \subseteq N_{g}$, i.e., $r \in\left(N_{g}: M_{g}\right)=A B$. Hence, $A B=\left(N_{e}: M_{e}\right)$. Since $M_{e}$ is multiplication, $N_{e}=\left(N_{e}: M_{e}\right) M_{e}=A B M_{e}=\left(A M_{e}\right)\left(B M_{e}\right)$ with $M_{e}=R_{e} M_{e}=(A+B) M_{e}=A M_{e}+B M_{e}$. Since $N_{e}$ is pseudo irreducible, either $A M_{e}=M_{e}$ or $B M_{e}=M_{e}$ and since $M_{e}$ is faithful, either $A=R_{e}$ or $B=R_{e}$.

Theorem 2.3. Let $R$ be a strongly $G$-graded ring and $M$ be a graded $R$-module. Suppose that $M_{e}$ is multiplication $R_{e}$-module and $N$ is a graded $R$-submodule of $M$. If $N_{e}$ is pseudo prime $R_{e}$-submodule of $M_{e}$, then $\left(N_{g}: M_{g}\right)$ is pseudo prime ideal of $R_{e}$ for all $g \in G$.

Proof. Let $g \in G$ and suppose that $I, J$ are two ideals of $R_{e}$ such that $I J \subseteq$ $\left(N_{g}: M_{g}\right)$ with $I+J=R_{e}$. Then $R_{g^{-1}} I M_{g}$ and $R_{g^{-1}} J M_{g}$ are $R_{e^{-}}$-submodules of $M_{e}$ such that $R_{g^{-1}} I M_{g}+R_{g^{-1}} J M_{g}=R_{g^{-1}}(I+J) M_{g}=R_{g^{-1}} R_{e} M_{g}=M_{e}$ and $\left(R_{g^{-1}} I M_{g}\right)\left(R_{g^{-1}} J M_{g}\right)=R_{g^{-1}} I J M_{g} \subseteq R_{g^{-1}} N_{g} \subseteq N_{e}$. Since $N_{e}$ is pseudo prime, either $R_{g^{-1}} I M_{g} \subseteq N_{e}$ or $R_{g^{-1}} J M_{g} \subseteq N_{e}$ and then either $I M_{g} \subseteq R_{g} N_{e} \subseteq$ $N_{g}$ or $J M_{g} \subseteq R_{g} N_{e} \subseteq N_{g}$, i.e., $I \subseteq\left(N_{g}: M_{g}\right)$ or $J \subseteq\left(N_{g}: M_{g}\right)$.

Theorem 2.4. Let $R$ be a strongly $G$-graded ring and $M$ be a graded $R$ module such that all components of $M$ are multiplication $R_{e}$-modules. Suppose that $N$ is a graded $R$-submodule of $M$. Then $N_{e}$ is pseudo prime $R_{e}$-submodule of $M_{e}$ if and only if $N_{g}$ is pseudo prime $R_{e}$-submodule of $M_{g}$ for all $g \in G$.

Proof. Suppose that $N_{e}$ is pseudo prime $R_{e}$-submodule of $M_{e}$. Let $g \in G$ and assume that $U, V$ are two $R_{e}$-submodules of $M_{g}$ such that $U V \subseteq N_{g}$ and $U+V=M_{g}$. Then $R_{g^{-1}} U$ and $R_{g^{-1}} V$ are $R_{e^{-}}$submodules of $M_{e}$ such that $\left(R_{g^{-1}} U\right)\left(R_{g^{-1}} V\right)=R_{g^{-1}} U V \subseteq R_{g^{-1}} N_{g} \subseteq N_{e}$ and $R_{g^{-1}} U+R_{g^{-1}} V=$ $R_{g^{-1}}(U+V)=R_{g^{-1}} M_{g}=M_{e}$. Since $N_{e}$ is pseudo prime, either $R_{g^{-1}} U \subseteq N_{e}$ or $R_{g^{-1}} V \subseteq N_{e}$ and then either $U \subseteq R_{g} N_{e} \subseteq N_{g}$ or $V \subseteq R_{g} N_{e} \subseteq N_{g}$. The converse is obvious. 
Theorem 2.5. Let $R$ be a strongly $G$-graded ring and $M$ be a graded $R$ module such that all components of $M$ are multiplication $R_{e}$-modules. Suppose that $N$ is a graded $R$-submodule of $M$. If $N_{e}$ is pseudo irreducible $R_{e}$-submodule of $M_{e}$, then $N_{g}$ is pseudo prime $R_{e}$-submodule of $M_{g}$ for all $g \in G$.

Proof. By Theorem 2.4, it is enough to prove that $N_{e}$ is pseudo prime. Let $U, V$ be two $R_{e}$-submodules of $M_{e}$ such that $U V \subseteq N_{e}$ and $U+V=M_{e}$. Then $U+N_{e}$ and $V+N_{e}$ are $R_{e}$-submodules of $M_{e}$ such that $\left(U+N_{e}\right)\left(V+N_{e}\right)=$ $U V+U N_{e}+V N_{e}+N_{e}^{2} \subseteq N_{e}$ and $\left(U+N_{e}\right)+\left(V+N_{e}\right)=(U+V)+N_{e}=M_{e}+N_{e}=$ $M_{e}$. Since $N_{e}$ is pseudo irreducible, either $U+N_{e}=M_{e}$ or $V+N_{e}=M_{e}$. If $U+N_{e}=M_{e}$, then $U+N_{e}=U+V$ and then $V \subseteq N_{e}$. Similarly, if $V+N_{e}=M_{e}$, then $U \subseteq N_{e}$.

An $R$-submodule $N$ of an $R$-module $M$ is said to be strictly non-prime if there exist $m \notin N$ and $r \notin(N: M)$ such that $r m \in N$ and $r M+R m=M$. An ideal of a ring $R$ is said to be strictly non-prime if there exist $a, b \notin I$ such that $a b \in I$ and $\langle a\rangle+\langle b\rangle=R$.

Theorem 2.6. Let $R$ be a strongly $G$-graded ring and $M$ be a graded $R$-module. Suppose that $M_{e}$ is multiplication $R_{e}$-module and $R_{e}$ is P.I.D. If $N$ is a graded $R$-submodule of $M$ such that $N_{e}$ is strictly non-prime $R_{e}$-submodule of $M_{e}$, then $\left(N_{g}: M_{g}\right)$ is strictly non-prime ideal of $R_{e}$ for all $g \in G$.

Proof. Since $N_{e}$ is strictly non-prime, there exist $m \notin N_{e}$ and $r \notin\left(N_{e}: M_{e}\right)$ such that $r m \in N_{e}$ and $r M_{e}+R_{e} m=M_{e}$. Since $M_{e}$ is multiplication, $R_{e} m=$ $I M_{e}$ for some ideal $I$ of $R_{e}$ and since $R_{e}$ is P.I.D, $I=\langle a\rangle$ for some $a \in R_{e}$. Now, let $g \in G$. Then if $a \in\left(N_{g}: M_{g}\right)$, then $I \subseteq\left(N_{g}: M_{g}\right)$ and then $I M_{g} \subseteq N_{g}$. So, $I M_{e}=I R_{g^{-1}} M_{g}=R_{g^{-1}} I M_{g} \subseteq R_{g^{-1}} N_{g} \subseteq N_{e}$ and then $R_{e} m \subseteq N_{e}$ a contradiction since $m \notin N_{e}$. So, $a \notin\left(N_{g}: M_{g}\right)$. Also, if $r \in\left(N_{g}: M_{g}\right)$, then $r M_{g} \subseteq N_{g}$ and then $r M_{e}=r R_{g^{-1}} M_{g}=R_{g^{-1}} r M_{g} \subseteq R_{g^{-1}} N_{g} \subseteq N_{e}$, i.e., $r \in\left(N_{e}: M_{e}\right)$ a contradiction. So, $r \notin\left(N_{g}: M_{g}\right)$. Now, $r a M_{g}=\operatorname{raR}_{g} M_{e}=$ $r R_{g} a M_{e} \subseteq r R_{g} I M_{e}=r R_{g} R_{e} m=R_{g} r m \subseteq R_{g} N_{e} \subseteq N_{g}$, i.e., $r a \in\left(N_{g}: M_{g}\right)$. Also, $M_{e}=r M_{e}+R_{e} m=r M_{e}+I M_{e}=r M_{e}+\langle a\rangle M_{e}$ and then $\langle r\rangle+\langle a\rangle=R_{e}$. Hence, $\left(N_{g}: M_{g}\right)$ is strictly non-prime ideal.

Theorem 2.7. Let $R$ be a strongly $G$-graded, $M$ be a graded $R$-module and $N$ be a graded $R$-submodule of $M$. Then $N_{e}$ is strictly non-prime $R_{e^{-}}$ submodule of $M_{e}$ if and only if $N_{g}$ is strictly non-prime $R_{e}$-submodule of $M_{g}$ for all $g \in G$.

Proof. Since $N_{e}$ is strictly non-prime, there exist $m \notin N_{e}$ and $r \notin\left(N_{e}: M_{e}\right)$ such that $r m \in N_{e}$ and $r M_{e}+R_{e} m=M_{e}$. Let $g \in G$. Then if $r \in\left(N_{g}: M_{g}\right)$, 
then $r M_{g} \subseteq N_{g}$ and then $r M_{e}=r R_{g^{-1}} M_{g}=R_{g^{-1}} r M_{g} \subseteq R_{g^{-1}} N_{g} \subseteq N_{e}$, i.e., $r \in\left(N_{e}: M_{e}\right)$ a contradiction. So, $r \notin\left(N_{g}: M_{g}\right)$. Suppose $R_{g} m \subseteq N_{g}$. Then $m=1 . m \in R_{e} m=R_{g^{-1}} R_{g} m \subseteq R_{g^{-1}} N_{g} \subseteq N_{e}$ a contradiction. So, $R_{g} m \nsubseteq N_{g}$. Now, $r R_{g} m=R_{g} r m \subseteq R_{g} N_{e} \subseteq N_{g}$ and also, $M_{g}=R_{g} M_{e}=R_{g}\left(r M_{e}+R_{e} m\right) \subseteq$ $R_{g} r M_{e}+R_{g} R_{e} m=r M_{g}+R_{e}\left(R_{g} m\right) \subseteq R_{e} M_{g}+R_{e}\left(R_{g} m\right)=M_{g}+R_{e}\left(R_{g} m\right) \subseteq$ $M_{g}+R_{e}\left(R_{g} M_{e}\right)=M_{g}+M_{g} \subseteq M_{g}$ and hence, $r M_{g}+R_{e}\left(R_{g} m\right)=M_{g}$. Therefore, $N_{g}$ is strictly non-prime. The converse is obvious.

\section{References}

[1] Z. Abd El-Bast, P.F. Smith, Multiplication modules, Comm. Algebra, 16, No. 4 (1988), 755-779.

[2] R. Ameri, On the prime submodules of multiplication modules, Inter. J. of Mathematics and Mathematical Sci., 27 (2003), 1715-1724.

[3] S. McAdam, R.G. Swan, Unique comaximal factorization, J. Algebra, 276, No. 1 (2004), 180-192.

[4] C. Nastasescu, F. Van Oystaeyen, Graded ring theory, Mathematical Library, 28, North Holland, Amesterdam (1982).

[5] M. Refai, Various types of strongly graded rings, Abhath Al-Yarmouk Journal (Pure Sciences and Engineering Series), 4, No. 2 (1995), 9-19. 
